\title{
Corresponsabilidad familiar, prácticas organizacionales, equilibrio trabajo-familia y bienestar subjetivo en Chile*
}

\author{
Family responsibility, organizational practices, work-family \\ balance and subjective welfare in Chile
}

Recibido: 25 de junio de 2014 - Revisado: 25 de septiembre de 2014 - Aceptado: 06 de noviembre de 2014

Andrés Jiménez Figueroa**

Verónica Gómez Urrutia ${ }^{* * *}$

\section{Resumen}

El estudio de la corresponsabilidad familiar ha adquirido una mayor relevancia en Chile. Investigaciones sugieren que desde los sectores público y privado se requieren distintas iniciativas orientadas hacia la implementación de políticas públicas que favorezcan esta inclusión. El panorama laboral mundial ha cambiado considerablemente en los ámbitos social, económico y familiar, exponiéndose la necesidad de reorganizar la distribución de las responsabilidades laborales entre hombres y mujeres. Como contribución a su discusión, se analiza aquí los principales antecedentes, y se plantea la necesidad de revisar las políticas públicas, aplicar las medidas en el ámbito organizacional e investigar nuevas medidas apropiadas a Chile como medios para mejorar la calidad de vida laboral en el país.

\section{Palabras clave}

Corresponsabilidad, trabajo-familia, políticas públicas.

\begin{abstract}
The study of family responsibility has become more relevant in Chile. Research suggests that from the public and private sectors various initiatives towards the implementation of public policies that favor this inclusion are required. The global labor scene has changed considerably in the social, economic and family areas, exposing the need to reorganize the distribution of work responsibilities between men and women. As a contribution to the discussion, we analyze here the main background, and the need to review public policies, to implement the measures in the organizational field and to investigate further appropriate measures to Chile as a means to improve the quality of working life in the country.
\end{abstract}

\section{Keywords}

Family responsibility, work-family, public policies.

\footnotetext{
* Artículo de revisión, derivado del Proyecto de Investigación de Calidad de Vida e Interfase Trabajo-Familia. Universidad de Talca - Universidad Autónoma, Talca, Chile.

** Psicólogo organizacional por la Universidad de Santiago de Chile, magíster en Administración y Dirección de Recursos Humanos (Chile) y doctorando en Administración de Empresas en Universidad Libre de Bruselas. Actualmente se desempeña como académico de la Facultad de Psicología e investigador en el Grupo de Investigación de Calidad de Vida y Ambientes Saludables, ambos pertenecientes a la Universidad de Talca, Chile. Correo electrónico: anjimenez@utalca.cl

*** Periodista por la Universidad de Chile, magíster en Ciencia Política por la Universidad Federal de Minas Gerais (Brasil) y doctora en Philosophy (Sociología) por la Universidad de Sussex (Reino Unido). En la actualidad se desempeña como académica de la Facultad de Ciencias Sociales y Humanidades e investigadora asociada al Centro de Estudios y Gestión Social del Maule, ambos pertenecientes a la Universidad Autónoma de Chile, sede Talca, Chile. Correo electrónico: gomezver@gmail.com
} 


\section{Introducción}

Durante las últimas décadas, el término corresponsabilidad ha adquirido una importancia creciente en los discursos sobre política social, tanto en el ámbito de la familia como en el del trabajo remunerado. La incorporación progresiva de la mujer al mercado de trabajo -que en Chile pasó de un $31 \%$ en 1990 a un $43 \%$ en 2011 (Ministerio de Desarrollo Social, 2011)-y la preocupación ascendente por la familia como institución han hecho de este un tema insoslayable. En una doble vertiente, se presenta por una parte la cuestión de la compatibilización de las responsabilidades familiares con el trabajo remunerado desde el punto de vista de la equidad de género (Organización Internacional del Trabajo, OIT, 2009, 2013); por otra, se enfatiza en el apoyo que deben entregar las organizaciones a sus trabajadores para facilitar esta adecuación (Jansen, Mohren, Van Amelsvoort, Janssen, \& Kant, 2010; Torío, Peña, Rodríguez, Fernández, \& Molina, 2010).

Los cambios en las estructuras sociales y económicas, así como en las expectativas de desarrollo de hombres y mujeres en Chile (Programa de las Naciones Unidas para el Desarrollo, PNUD, 2010) han llevado a que ambos sexos se integren al mercado laboral (Riquelme, Rojas, \& Jiménez, 2012). No obstante, ello no ha sido acompañado de rupturas significativas en las concepciones culturales predominantes, según las cuales el trabajo reproductivo -que comprende el trabajo doméstico y el cuidado de dependientes $^{1}$ (Razavi, 2007)- es una responsabilidad de las familias (y más específicamente, de las mujeres), y no una necesidad de las sociedades. Este tipo de labor ha sido tradicionalmente visto como un bien "privado", algo que las familias producen para sí mismas y que, por lo tanto, no tendría relación con el trabajo remunerado (Fineman, 2004).

Dicha mirada ha sido objeto de problematización en la medida en que se cuestiona la idea de una vida centrada en el trabajo (Organisation for Economic Cooperation and Development, OECD, 2011) y la división sexual de tareas, que coloca a las mujeres como principales responsables del ámbito doméstico y de cuidado.

Estos cambios abren una oportunidad para el surgimiento de nuevas formas de organización de la vida familiar y laboral (Brullet, 2010). Tal reconstrucción reclama la reorganización de los roles entre hombres y mujeres, buscando un mayor equilibrio en el compromiso de la pareja sobre los tiempos destinados a distintos quehaceres en la vida familiar, tales como los oficios domésticos, soporte económico, la educación y el cuidado de los hijos (Torío et al., 2010). En este contexto, el presente trabajo procura aportar algunas claves conceptuales para situar la corresponsabilidad familiar en la discusión sobre las posibilidades de reorganizar la relación entre trabajo y familia, considerando el rol crucial del Estado, las instituciones y los propios trabajadores en esta tarea (Balmforth \& Garden, 2006).

\section{Situando el problema: el conflicto trabajo-familiaylacorresponsabilidad}

Las mujeres están entrando en números cada vez mayores al mercado laboral, a la política y a otros espacios que por tradición se consideran masculinos. No obstante, el movimiento inverso ha ocurrido con mucho menos fuerza. Si bien sondeos de opinión recientes sugieren que los hombres desean tener un rol más activo en la crianza de los hijos ${ }^{2}$, la incorporación efectiva de los varones a la ejecución de labores domésticas y de cuidado no se ha producido masivamente, resultando muchas veces en una sobrecarga de trabajo femenino (Junta Nacional de Jardines Infantiles -Junji-, Organización de las Naciones Unidas para la Educación, la Ciencia y la Cultura -Unesco-, \& Fondo de las Naciones Unidas para la Infancia-Unicef-, 2010; PNUD, 2010) y en la falta de desarrollo de habilidades de crianza en los hombres (Kershaw, 2005).

En este sentido, la cuestión de la conciliación trabajo-familia se hace evidente en tanto 
que la división de tareas por sexo, que asignaba a las mujeres el papel de cuidadoras y a los varones, el de proveedores, se revela como uno de los "núcleos duros" de la desigualdad de género (OIT, 2013), ya que merma las posibilidades de desarrollo laboral y económico de las mujeres, y de desarrollo emocional para los varones. El conflicto trabajo-familia se refleja -entre otros fenómenos- en la prolongada ausencia de los progenitores en el hogar y en la insuficiencia de habilidades y destrezas para conciliar las diferentes esferas y roles sociales (Godoy \& Mladinic, 2009; Riquelme et al., 2012), trayendo como consecuencia preocupaciones incompatibles en ambas esferas (Greenhaus \& Beutell, 1985; Otálora, 2007). El fenómeno conocido como spillover, que se caracteriza por el desequilibrio en el tiempo dedicado a cada uno de estos ámbitos, determina de manera importante que uno acaba afectando al otro (Repetti, 1987; Román, Avendaño, \& Tomicic, 2004; Ugarteburu, Cerrato, \& Ibarretxe, 2008).

En este escenario, nivelar las demandas por tiempo y energía en cada uno de estos ámbitos, así como los grados de compromiso psicológico y de satisfacción en cada uno de ellos, es decisivo para el bienestar personal de los trabajadores y las trabajadoras (Greenhaus, Collins, \& Shaw, 2003). En otras palabras, en la medida en que se cuestionan los roles tradicionales de género, en particular entre las generaciones más jóvenes (Aguayo et al., 2011; Pontificia Universidad Católica de Chile \& Adimark, 2008), el conflicto trabajofamilia aparece como una cuestión de justicia y equidad, pero también de bienestar subjetivo, entendido como la manera en que las personas piensan y sienten con respecto a sus vidas y a las conclusiones, tanto cognitivas como afectivas, que alcanzan cuando evalúan su existencia (Cuadra \& Florenzano, 2003). Estos autores dan a conocer también dos consensos en relación con el concepto: el primero se asocia con la existencia de una dimensión básica y general de carácter subjetivo, mientras que el segundo plantea que el bienestar se compone de dos facetas elementales, donde una se centra en los aspectos afectivo-emocionales y la otra en los aspectos cognitivo-valorativos. Ambas son fundamentales para el ajuste psicológico de los individuos, tanto en el hogar como en el trabajo.

Debeljuh y Jáuregui (2004) proponen tres pilares para equilibrar las demandas del trabajo y de la familia: el trabajador, el Estado y la empresa. En cuanto a la labor del trabajador, este debe implementar sus propias estrategias, que le permitan alcanzar objetivos realistas con expectativas familiares y laborales claras, fomentando respeto mutuo y una distribución equitativa de poder y responsabilidades. Por su parte, la empresa es quien se ocupa de asegurar el bienestar de sus empleados y de crear un ambiente laboral que posibilite el equilibrio en ambas áreas. Finalmente se encuentra el Estado, quien debe velar por los intereses de las familias, diseñando marcos legales y regulatorios, tales como las políticas laborales, que favorezcan el equilibrio trabajo-familia en un escenario de equidad.

La conceptualización de esta tríada como cimiento para enfrentar el conflicto trabajofamilia se refleja en la idea de corresponsabilidad familiar, entendida como un principio de organización de la energía, tiempo y desarrollo de habilidades que resultan determinantes en una familia para llevar a cabo las tareas del hogar y el cuidado de los hijos (Maganto, Etxeberría, \& Porcel, 2010; Rodríguez, Peña, \& Torío, 2010). Así, el término se refiere a la necesidad de reorganizar la distribución de responsabilidades laborales y familiares entre hombres y mujeres, así como también entre familias, Estado, mercado y sociedad en general (OIT, 2009), de manera que todas las personas puedan potenciar cada área de su vida de modo autónomo y armónico. Según la OIT y el PNUD (2009): "La aspiración de una agenda de conciliación con corresponsabilidad debe ser el ampliar los grados de libertad, para que hombres y mujeres puedan elegir distintas formas de combinación entre vida laboral y familiar" (p. 18). 
Desde la perspectiva de los individuos, la familia es sin duda un lugar de refugio emocional y afecto, pero puede ser también una fuente de tensión en tanto que las exigencias propias de la vida familiar se perciban como inequitativas o como entrando en conflicto con el mundo del trabajo remunerado (Maganto et al., 2010; Organización de las Naciones Unidas, ONU, 2007; PNUD, 2010). En ese marco, las estrategias singulares que hombres y mujeres puedan desarrollar para enfrentar el conflicto trabajo-familia dependerán de las capacidades de negociación con que cuenten tanto en el hogar como en la labor remunerada.

Dentro de las variables involucradas en la distribución de roles entre hombres y mujeres en la familia, se observa que el poder dentro de la pareja se halla fuertemente influenciado por los aspectos económicos. Así, la teoría de los recursos argumenta que el cónyuge que posee más recursos, es quien tiene más poder sobre la familia, de forma que mantiene menos implicación en el trabajo parental (Rodríguez et al., 2010). Desde el punto de vista de la teoría de género, dados los diferenciales de poder económico (acceso al trabajo remunerado) y simbólico (roles de género) que existen entre hombres y mujeres, estas últimas contarían con un margen de negociación menor. Por ejemplo, $\mathrm{y}$ en cuanto al poder simbólico, según la Encuesta de Caracterización Socioeconómica Nacional (Ministerio de Desarrollo Social, 2011), entre las mujeres que no están trabajando o están buscando trabajo, casi el $50 \%$ indicó razones vinculadas a las tareas domésticas o al cuidado de dependientes, mientras solo el 10,5 $\%$ de los varones en las mismas circunstancias señaló este tipo de razones. Y de acuerdo con un estudio del Servicio Nacional de la Mujer (Sernam) (2010), las mujeres perciben menos apoyo de sus parejas en los cuidados de los menores (un $9 \%$ frente a un $39 \%$ de los hombres), sugiriendo la persistencia de patrones culturales que caracterizan a las mujeres como responsables de la crianza, el ámbito doméstico y el cuidado de dependientes.
De igual manera, diferencias de ingreso entre hombres y mujeres y entre trabajadores/as de una misma empresa- inciden en las capacidades personales para la negociación de espacios de conciliación entre trabajo y familia y, con ello, en la posibilidad de evaluar de modo positivo el propio bienestar. Datos de la Encuesta Nacional de la Primera Infancia (Junji et al., 2010) apuntan que las probabilidades de ejercer efectivamente derechos ya adquiridos en el contexto de la familia (como los permisos parentales), varían por sexo y estrato socioeconómico, mostrando las limitaciones que la búsqueda de estrategias individuales de conciliación presenta.

Desde este punto de vista, la búsqueda del equilibrio trabajo-familia contiene una dimensión individual, dada por la capacidad de implementar estrategias individuales para concertar ambos ámbitos, lo cual incidiría en la percepción del propio bienestar. Al mismo tiempo, existe una importante dimensión social, ya que algunas de las cortapisas a la puesta en marcha de estrategias personales provienen de la forma misma en que se estructura el trabajo remunerado -con largas jornadas laborales y poca flexibilidad horaria- y también el no remunerado, en términos de los roles de género que plantean a las mujeres como principales responsables del buen funcionamiento cotidiano de la familia. Reca (2008) puntualiza que en Chile el debate público aún se sitúa entre la idea de flexibilizar el empleo para permitir que las mujeres continúen desempeñando sus roles tradicionales, y la demanda por una mirada más amplia que implique repensar la relación familiatrabajo y la división sexual tradicional de tareas dentro y fuera del hogar (Todaro, 2006). De allí la necesidad de considerar en el análisis a los pilares institucionales, las empresas y el Estado, examinados en la sección siguiente.

Los pilares institucionales: políticas y programas que incorporan conciliación trabajofamilia

Junto a las implicaciones que tiene la corresponsabilidad para la justa redistribución de 
los roles de la familia, es significativo considerar cómo las instituciones se hacen cargo de estas temáticas por medio de la implementación de prácticas organizacionales que van en beneficio de la conciliación de la vida familiar y laboral (Caballer, Peiró, \& Sora, 2011). El apoyo organizacional puede ser clave para que las personas que trabajan puedan distribuir de mejor manera los tiempos dedicados a cada una de las esferas, familia y trabajo.

En estudios realizados utilizando el modelo de cultura trabajo-familia (Allen, 2001; Thompson, Beauvais, \& Lyness, 1999), se observa que la percepción de apoyo, tiene un impacto positivo en las actitudes del trabajador hacia su empleo, en especial sobre el compromiso, y más específicamente, sobre el compromiso afectivo (Jiménez, Acevedo, Salgado, \& Moyano, 2009). En este sentido, la literatura señala como factores importantes los niveles de apoyo organizacional percibidos y una cultura de empresa que pueda transferir los valores, metas y normas -orientados a la conciliación- a todos los niveles de la institución, proporcionando en efecto el apoyo necesario a los trabajadores para que sientan satisfacción tanto por su vida laboral como por su vida en familia (Caballer et al., 2011; Tejada \& Arias, 2005). En definitiva, una labor remunerada que permite a las personas una mayor autonomía y márgenes de discrecionalidad acerca de cómo y cuándo se hace el trabajo, permite a los empleados cumplir mejor las múltiples demandas que conlleva el vínculo trabajo-familia (Thompson \& Prottas, 2005).

No obstante, medidas tales como horarios de trabajo flexibles, referencias de cuidado infantil y servicios de asistencia directa poseen una historia reciente en la planificación de gobiernos e instituciones privadas y públicas (Franco \& Sánchez, 2008; OIT \& PNUD, 2009). Bosch, Cano, Riumalló y Tarud (2014) realizaron un estudio sobre esta materia en entidades públicas y privadas chilenas, incluyendo empresas de diferentes rubros. Con soporte en consultas sistemáticas a directivos, personal de recursos humanos y sindicalistas/dirigentes de estas organizaciones, los autores constataron que el $61 \%$ de los encuestados no conocía el concepto de conciliación con corresponsabilidad como mecanismo de compatibilización entre trabajo y familia. Y aunque se atribuye gran valor a la familia y a los roles parentales, se reconoce al mismo tiempo una cultura del empleo que premia la permanencia, durante largas horas, en el lugar de trabajo. Las políticas de conciliación (en particular con corresponsabilidad) son vistas como costosas y difíciles de controlar (Bosch et al., 2014), particularmente las de flexibilidad horaria.

En la misma vertiente, según la investigación hecha en 2013 por la Fundación Chile Uni$\mathrm{do}^{3}$, hay consenso en los colaboradores de las 114 organizaciones consultadas (que incluyeron organizaciones públicas y privadas de diversos tamaños) respecto a que la flexibilidad en el horario de trabajo sobre la base del cumplimiento de objetivos o trabajar desde la casa, es el tipo de medida a la que se asigna mayor prioridad $y$, de forma paradójica, una de las que menos se implementan. Así mismo, existe la idea de que estas medidas se dirigen, principalmente, a las mujeres, y no a todas las personas que trabajan.

Las políticas del entorno privado que se encaminan hacia la conciliación entre el ámbito laboral y la vida familiar son instrumentos fundamentales para generar estabilidad en las organizaciones, y mecanismos que posibilitan una participación más equitativa entre hombres y mujeres en el mercado laboral (Arroyo, 2007). También proporcionan a los empleados recursos para apoyar la gestión de las responsabilidades laborales y familiares (Casper, Harris, TaylorBianco, \& Holliday, 2011; Thompson \& Prottas, 2005). En el mismo eje, las políticas públicas y la legislación laboral son una herramienta privilegiada para promover cambios en las formas en que las propias familias distribuyen las tareas al interior del hogar, por lo cual pueden propiciar una mayor equidad de género dentro y fuera del ámbito del trabajo remunerado. 
En esa línea, en 2006 el Sernam lanzó un Código de buenas prácticas laborales y no discriminación, como parte del cumplimiento de las 36 medidas prioritarias para los cien primeros días de la primera administración de la presidenta Michelle Bachelet (2006-2010). El texto fomenta la paridad de género y las relaciones igualitarias entre hombres y mujeres, proponiendo políticas para mejorar el acceso, la capacitación y el desarrollo profesional, conciliando la vida laboral y familiar. La adhesión a las normas del código da a las organizaciones públicas y privadas la opción de obtener una certificación (el "Sello Iguala") en la norma chilena NCh 3262. Sobre la base de este código, la Confederación de la Producción y del Comercio (CPC) adoptó ese mismo año una "Guía de buenas prácticas laborales sobre no discriminación en la empresa", que incluye una sección sobre "Conciliación de responsabilidades laborales con obligaciones familiares", en la que se recomienda a las compañías impulsar una cultura destinada a compatibilizar las responsabilidades laborales y parentales de hombres y mujeres, así como disponer de mecanismos alternativos de distribución de jornada, que faciliten el cumplimiento de las obligaciones familiares de ambos progenitores, en la medida que ello sea compatible con las necesidades de la empresa.

No obstante, y en contraste con los principios declarados, la legislación laboral vigente en el país solo considera las responsabilidades familiares en el embarazo/nacimiento y en caso de enfermedad del hijo menor de un año, con tímidos intentos de alterar la división sexual del trabajo en las responsabilidades parentales: la ley 20.047 de 2005 establece un permiso pagado de cinco días para los varones en caso de nacimiento de un hijo o una hija. Y el posnatal se extendió en 2011, incorporando al régimen existente de descanso maternal -seis semanas de prenatal y 12 de posnatal- un permiso posnatal parental de 12 semanas, que amplía el descanso de maternidad a seis meses, con un subsidio equivalente al $100 \%$ del salario, hasta un tope de USD 3.000 dólares mensuales (ley
20.545 de 2011), e introduciendo la posibilidad de que una parte del posnatal se transfiera al padre, por decisión de la madre. Sin embargo, en un contexto de jornadas laborales largas y significativas brechas salariales por género, es poco probable que estas medidas, por sí mismas, promuevan la corresponsabilidad familiar.

\section{Conclusiones}

Las políticas de conciliación trabajo-familia aparecen como un factor muy importante en el logro de objetivos socialmente relevantes, como la mejora de la calidad de vida familiar. Empero, focalizar el debate sobre conciliación en los individuos y sus familias (y principalmente, en las mujeres), en el entendido de que se trata de un problema privado, obscurece la desigual distribución de recursos y oportunidades que hombres y mujeres tienen para concertar las diferentes dimensiones de sus vidas $\mathrm{y}$, con ello, de alcanzar el necesario equilibrio entre los ámbitos del trabajo y la familia.

En esa línea, la demanda por una mirada más amplia sobre el problema implica repensar la relación familia-trabajo como una cuestión que compete a toda la sociedad. Si ello no ocurre y las obligaciones familiares se plantean como compitiendo con las oportunidades laborales, es muy probable que se agudice el desequilibrio entre ambas esferas, así como el reparto dispar de oportunidades de vida entre hombres y mujeres y el consiguiente impacto en el bienestar subjetivo de todos los trabajadores y trabajadoras.

En el caso de Chile, hemos argumentado, la legislación vigente incorpora tímidamente a los padres en los derechos asociados a la esfera familiar, en especial en el cuidado de hijos e hijas recién nacidos; las organizaciones, por su parte, han realizado escasos avances hacia una política de corresponsabilidad, en términos de promover activamente la incorporación de los hombres a la esfera doméstica y del cuidado (Fundación Chile Unido, 2013). Y si bien estas 
políticas inciden en los recursos y capacidades de negociación de espacios de concertación entre trabajo y familia con que cuentan hombres y mujeres, la persistencia de patrones tradicionales sugiere que, en tanto que no se tienda a la armonía entre oportunidades y recompensas entre estos dos universos, el trabajo con menor reconocimiento seguirá siendo efectuado por los grupos más desfavorecidos en la sociedad -en el caso que nos ocupa, por las mujeres (Kershaw, 2006)-. Desde este punto de vista, estimular la conciliación con corresponsabilidad aparece también como una ocasión para incrementar la autonomía y bienestar de las personas.

En materia organizacional se constata que no basta con proveer de un conjunto de políticas o prácticas organizacionales dirigido a respaldar el equilibrio trabajo-familia de sus trabajadores, si no existe una cultura y un efectivo apoyo directivo de tales medidas. El reto de la conciliación trabajo-familia es un tema complejo que exige integrar estrategias individuales/familiares, organizacionales y políticas públicas (Cantera, Cubells, Martínez, \& Blanch, 2009). En este marco, existe abundante evidencia internacional que enfatiza en los beneficios -tanto para el empleado como para la empresa- de políticas de conciliación trabajofamilia (mayor compromiso organizacional, menor rotación en el empleo, mejor calidad de vida laboral). Sin embargo, en Chile se dispone todavía de poca evidencia empírica que permita sustentar con solidez una conclusión similar, lo cual constituye una limitación para este estudio. Por ello, resulta necesaria más investigación en esta temática; en específico acerca de las barreras percibidas para la implementación de las políticas y medidas que favorezcan la armonía trabajo-familia y la corresponsabilidad. De acuerdo con López-Ibor, Escot, Fernández y Palomo (2010) y Caunedo \& Rodríguez (2008), estas pueden agruparse en tres grandes áreas:

a) Barreras estructurales, tales como clase de actividad productiva (proceso, tipo de horarios, maquinaria, etc.) o bien el horario en el que se realiza, que dificulten la adopción de medidas de conciliación. Aquí se incluyen las exigencias de movilidad laboral y la precariedad del empleo, que limita el poder de negociación ante la empresa.

b) Barreras culturales (ideológicas), referidas a las representaciones tradicionales sobre género y familia (hombres proveedores, mujeres cuidadoras); posibles diferencias en la valoración de las familias, y la lectura que en la empresa se hace del disfrute de medidas de conciliación, en términos de considerarla un costo para la compañía o, por el contrario, una forma de fidelizar a la fuerza de trabajo, y

c) Barreras políticas, atinentes a la percepción de las dificultades u oportunidades que provee el entorno -por ejemplo, la legislación, la política tributaria o el mercado laboral-.

La identificación explícita de estos obstáculos permitiría establecer una agenda para la conciliación trabajo-familia que considere la tríada que ya hemos identificado en este artículo -trabajadores, empresas y Estado- como actores centrales de un proceso de reorganización de la vida familiar y laboral. En la medida en que esta agenda construya puntos de convergencia y se aplique, podría ampliar los márgenes de negociación de los actores. Y, en el mediano plazo, permitiría evaluar la eficacia de este tipo de medidas en el contexto chileno.

\section{Notas}

${ }^{1}$ El concepto de trabajo reproductivo se refiere a las actividades necesarias para la reproducción cotidiana de la sociedad: trabajo doméstico y cuidado de niños/as, adultos mayores y personas con necesidades especiales o enfermedades crónicas.

${ }^{2}$ El estudio de Aguayo, Correa y Cristi (2011), basado en una encuesta representativa para Santiago, Valparaíso y Concepción, reporta que ocho de cada diez hombres que viven con 
sus hijos y trabajan $(75,9 \%)$ indicaron que les "gustaría trabajar menos si eso significara pasar más tiempo con [sus] hijos”. Un 61,7 \% señaló que "dedican muy poco tiempo a sus hijos por motivos de trabajo".

${ }^{3}$ La Fundación Chile Unido otorga el premio "Mejores empresas para padres y madres que trabajan", para lo cual realiza un estudio entre las compañías participantes. Por tratarse de un premio al que las organizaciones se presentan (autoselección), utilizamos este estudio solo a modo de ilustración.

\section{Referencias}

Aguayo, F., Correa, P., \& Cristi, P. (2011). Encuesta IMAGES Chile. Resultados de la encuesta internacional de masculinidades y equidad de género.Santiago de Chile: CulturaSalud; EME.

Allen, T. (2001). Family-Supportive Work Environments: The role of organizational perceptions. Journal of Vocational Behavior, 58, 414-435.

Arroyo, C. (2007). Perspectivas laborales en torno a la conciliación de la vida familiar y laboral: el reto de la compatibilidad y la corresponsabilidad. Anuario Jurídico y Económico Escurialense, 40, 243-270.

Balmforth, K., \& Garden, D. (2006). Conflict and facilitation between work and family: realizing the outcomes for organizations. New Zealand Journal of Psychology, 35(2), 69-7.

Bosch, M., Cano, V., Riumalló, M., \& Tarud, C. (2014). Estudio Percepciones y Prácticas de Conciliación y Corresponsabilidad en Organizaciones Públicas y Privadas. Recuperado de http://www.ese.cl/publicaciones/estudio-percepciones-y-practicas-de-conciliacion-y-corresponsabilidaden-organizaciones-publicas-y-privadas/
Brullet, C. (2010). Cambios familiares y nuevas políticas sociales en España y Cataluña. El cuidado de la vida cotidiana a lo largo del ciclo de vida. Educar, 45, 51-79.

Caballer, A., Peiró, J. M., \& Sora, B. (2011). Consecuencias de la inseguridad laboral. El papel modulador del apoyo organizacional desde una perspectiva multinivel. Psicothema, 23(3), 394-400.

Cantera, L., Cubells, M., Martínez, L., \& Blanch, J. (2009). Work, Family, and Gender: Elements for a Theory of Work-Family Balance. The Spanish Journal of Psychology, 12, 641-647.

Casper, W., Harris, C., Taylor-Bianco, A., \& Holliday, J. (2011).Work-family conflict, perceived supervisor support and organizational commitment among Brazilian professionals. Journal of Vocational Behavior, 79, 640-652.

Caunedo, P., \& Rodríguez, A. (2008). De la conciliación a la corresponsabilidad: buenas prácticas y recomendaciones. Madrid: Instituto de la Mujer.

Cuadra, H., \& Florenzano, R. (2003). El bienestar subjetivo: hacia una psicología positiva. Revista de Psicología de la Universidad de Chile, 12(1), 83-96.

Debeljuh, P., \& Jáuregui, K. (2004). Trabajo y Familia. Hacia una cultura familiar amigable en el contexto latinoamericano. Esan-cuadernos de difusión, 16, 91-102.

Fineman, M. (2004). The Autonomy Myth. A Theory of Dependency. Nueva York: The New Press.

Franco, S. M., \& Sánchez, G. (2008). Las familias: ¿Un asunto de políticas públicas? Revista Sociedad y Economía, 14, 82-10. 
Fundación Chile Unido. (2013). Mejores empresas para madres y padres que trabajan. Recuperado de http://www.chileunido.cl/wp-content/uploads/2013/01/ Informe-General-2012.pdf

Godoy, L., \& Mladinic, A. (2009). Estereotipos y Roles de Género en la Evaluación Laboral y Personal de Hombres y Mujeres en Cargos de Dirección. PSYKHE, 18(2), 51-64.

Greenhaus, J., \& Beutell, N. (1985). Sources of conflict between work and family roles. Academy of Management Review, 10, 7688.

Greenhaus, J., Collins, K., \& Shaw, J. (2003). The relation between work- family balance and quality of life. Journal of Vocational Behavior, 63, 510- 531.

Jansen, N., Mohren, D., Van Amelsvoort, L., Janssen, N., \& Kant, I. (2010). Changes in working time arrangements over time as a consequence of work-family conflict. Chronobiology International, 27(5), 1045-1061.

Jiménez, A., Acevedo, D., Salgado, A., \& Moyano, E. (2009). Cultura trabajo- familia y compromiso organizacional en empresa de servicios. Psicología em estudo, 14(4), 729-738.

Jiménez, A., \& Moyano, E. (2008). Factores laborales de equilibrio entre trabajo y familia: medios para mejorar la calidad de vida. Revista Universum, 23(1), 116-133.

Junta Nacional de Jardines Infantiles -[JUNJI]., Organización de las Naciones Unidas para la Educación, la Ciencia y la Cultura -[UNESCO]., \& Fondo de Naciones Unidas para la Infancia -[Unicef]. (2010). Encuesta Nacional de la Primera Infancia, ENPI 2010 - Resultados Preliminares. Santiago de Chile: Autores. Re- cuperado de http://web.integra.cl/doctos cedoc/archivos/documentos/Informe $\% 20$ Final\%20Preliminar\%20ENPI.pdf

Kershaw, P. (2005). Carefair: Rethinking the Responsibilities and Rights of Citizenship. Vancouver: University of British Columbia Press.

Kershaw, P. (2006). Care fair: Choice, Duty, and the Distribution of Care. Social Politics: International Studies in Gender, State and Society, 13(3), 341-371.

López-Ibor, R., Escot, L., Fernández, J., \& Palomo, M. (2010). Las politicas de conciliación de la vida familiar y laboral desde la perspectiva del empleador. Madrid: Universidad Complutense de Madrid.

Maganto, M., Etxeberría, J., \& Porcel, A. (2010). La corresponsabilidad entre los miembros de la familia, como factor de conciliación. Educatio Siglo XXI, 28(1), 69-84.

Organisation for Economic Cooperation and Development -[OECD]. (2011). Doing Better for Families. Recuperado de http:// dx.doi.org/10.1787/9789264098732-en

Organización de las Naciones Unidas -[ONU]. (2007). Objetivos de Desarrollo del Milenio. Informe 2006 una mirada a la igualdad entre los sexos y la autonomía de la mujer en América Latina y el Caribe. Santiago de Chile: Autor.

Organización Internacional del Trabajo -[OIT]. (2013). Trabajo decente y cuidado compartido. Hacia una propuesta de parentalidad. Santiago de Chile: Autor; Programa de las Naciones Unidas para el Desarrollo -[PNUD].

Organización Internacional del Trabajo -[OIT]., \& Programa de las Naciones Unidas para 
el Desarrollo -[PNUD]. (2009). Trabajo y familia: Hacia nuevas formas de conciliación con corresponsabilidad social. Santiago: Autor.

Otárola, G. (2007). La relación existente entre el conflicto trabajo- familia y el estrés individual en dos organizaciones colombianas. Cuadernos de Administración, 20 (34), 139- 160.

Pontifica Universidad Catóica de Chile., \& Adimark. (2008). Encuesta Bicentenario. Recuperado de http://www7.uc.cl/encuestabicentenario/encuestas/2008/index.html

Programa de las Naciones Unidas para el Desarrollo -[PNUD]. (2010). Desarrollo Humano en Chile: Género, los desafios de la Igualdad. Santiago de Chile: Autor.

Razavi, S. (2007). The Political and Social Economy of Care in a Development Context. Conceptual Issues, Research Questions and Policy Options (United Nations Research Institute for social Development -UNRISD, Gender and Development Programme Paper Number 3). Ginebra: UNRISD.

Reca, I. (2008). Politicas para la conciliación de las responsabilidades laborales y familiares en Argentina, Chile, Paraguay y Uruguay. Santiago: Programa de la Naciones Unidas para el Desarrollo [PNUD]; Organización Internacional del Trabajo -[OIT].

Repetti, R. (1987). Individual and common components of the social environment. Journal of Personality and Social Psychology, 52, 710- 720.

República de Chile. Ley 20.047 de 2005 . Establece un permiso paternal en el Código del Trabajo. Diario Oficial de la Repúbli- ca de Chile. Ministerio del trabajo y Previsión Social, septiembre de 2005

República de Chile. Ley 2.545 de 2011. Modifica las normas sobre protección a la maternidad e incorpora el permiso postnatal parental. Diario Oficial de la República de Chile. Ministerio del trabajo y Previsión Social, octubre de 2011.

República de Chile. Ministerio de Desarrollo Social. (2011). Resultados de la encuesta de caracterización Socioeconómica Nacional 2011. Módulo Mujer y Familia. Recuperado de http://centrodelafamilia. uc.cl/201304043080/familia-chilena/ resultados-mujer-y-familia-casen-2011. html

Riquelme, E., Rojas, A., \& Jiménez, A. (2012). Equilibrio trabajo-familia, apoyo familiar, autoeficacia parental y funcionamiento familiar percibidos por funcionarios públicos de Chile. Trabajo y Sociedad, 18, 203-215.

Rodríguez, M., Peña, J., \& Torío, S. (2010). Corresponsabilidad familiar: negociación e intercambio en la división del trabajo doméstico. Papers, 95(1), 95-117.

Román, J., Avendaño, C., \& Tomicic, A. (2004). Conflicto y equilibrio en la relación trabajo familia: Un estudio con mujeres trabajadoras por cuenta propia. Persona y Sociedad, 18(1), 51-76.

Servicio Nacional de la Mujer -[SERNAM]. (2010). Encuesta de conciliación laboralfamiliar ISUC-SERNAM. Recuperado de http://estudios.sernam.cl/documentos/? eMjI4OTQ3NA==-Encuesta Conciliaci $\% \mathrm{C} 3 \% \mathrm{~B} 3 \mathrm{n} \_$Laboral-Familiar

Tejada, J. M., \& Arias, F. (2005). Prácticas organizacionales y el compromiso de los 
trabajadores hacia la organización. Enseñanza e Investigación en Psicología, 10(2) 295-309.

Thompson, C., \& Prottas, D. (2005). Relationships among organizational family support, job autonomy, perceived control and employee well-being. Journal of Occupational Health Psychology, 10(4), 100-118.

Thompson, C. A., Beauvais, L. L., \& Lyness, K. S. (1999). When work-family benefits are not enough: The influence of work-family climate on benefit utilization, organizational attachment, and work-family conflict. Journal of Vocational Behavior, 54, 392-415.
Todaro, R. (2006). ¿Flexibilidad laboral o precarización? El debate sobre la reproducción social. En C. López, A. Espino, R. Todaro \& N. Sanchís, América Latina, un debate pendiente (pp. 131-162). Montevideo: REPEM; DAWN; IFC.

Torío, S., Peña, J.C., Rodríguez, M.C., Fernández, C.M., \& Molina, S. (2010). Hacia la corresponsabilidad familiar: Construir lo cotidiano. Un programa de educación parental. Educatio Siglo XXI, 28(1), 85-108.

Ugarteburu, I., Cerrato, J., \& Ibarretxe, R. (2008). Transformando el Conflicto Trabajo/Familia en Interacción y Conciliación Trabajo/ Familia. Lan Harremanak. Revista de Relaciones Laborales. 18, 17-41. 
